Meta

Journal des traducteurs

Translators' Journal

\title{
La terminologie criminologique : un instrument et un symptôme
}

\section{Jacqueline De Plaen}

Volume 24, numéro 1, mars 1979

La traduction juridique

URI : https://id.erudit.org/iderudit/003841ar

DOI : https://doi.org/10.7202/003841ar

Aller au sommaire du numéro

Éditeur(s)

Les Presses de l'Université de Montréal

ISSN

0026-0452 (imprimé)

1492-1421 (numérique)

Découvrir la revue

Citer cet article

De Plaen, J. (1979). La terminologie criminologique : un instrument et un symptôme. Meta, 24(1), 141-145. https://doi.org/10.7202/003841ar d'utilisation que vous pouvez consulter en ligne. 


\title{
La terminologie criminologique : un instrument et un symptôme
}

\author{
\&... il y a deux sortes d'universitaires : les humanistes, les \\ littéraires qui ne connaissent rien à la technologie, et les \\ scientifiques qui se moquent pas mal de la langue. » \\ Eugène Ionesco
}

Où se situent, entre ces deux pôles, les sciences dites humaines et quelle est leur attitude face à la langue ? Là où le chimiste et le physicien disposent d'un langage universel et univoque par excellence, celui des chiffres et des symboles, le criminologue, pour ne parler que de lui, doit travailler avec des mots. Le langage technique de l'industrie, le vocabulaire administratif et commercial véhiculent quantité de néologismes, importent des États-Unis ou d'ailleurs, quantité de termes sur le sort desquels des services linguistiques se penchent avec attention. Le Comité d'étude des termes techniques français, par exemple, constitue selon les dires de son président, $M$. Combet, un véritable «bureau d'immigration pour ces termes $»$. On en examine la fertilité, on étudie leurs racines grecques ou latines et les possibilités de leur adjoindre des préfixes ou suffixes. Les normes d'acceptation des mots techniques importés sont aussi bien définies : clarté, fécondité et internationalisation. Il s'agit là de domaines bien concrets et les échanges internationaux créent une demande toujours plus pressante. Aussi existe-t-il de nombreux lexiques techniques; l'UNESCO et les gouvernements publient par exemple des dictionnaires et des glossaires polyglottes sur les sujets les plus divers tels que les savons, le pétrole, la pêche commerciale et sportive.

Comment expliquer la pénurie et les lacunes des dictionnaires touchant les sciences sociales et la criminologie en particulier? La langue n'est-elle pas le premier et presque le seul outil du criminologue? Celui-ci ne subit-il pas autant que ses collègues des sciences exactes les influences venues de l'étranger? Il est vrai que le problème de traduction qui se pose dans les domaines techniques se complique, en ce qui concerne les sciences sociales, du fait qu'elles sont multidisciplinaires, qu'elles font appel à plusieurs niveaux de langue à la fois et qu'elles évoluent avec une rapidité foudroyante.

En criminologie, on doit remonter à 1960 pour trouver l'Elsevier's Dictionary of Criminal Science. Cet ouvrage, le premier du genre, traduit en 8 langues (anglais, français, allemand, néerlandais, italien, espagnol, portugais et suédois) quelque 10930 termes. De consultation facile, il précise pour chaque mot de quelle science ou technique il relève et en fournit, à l'occasion, les équivalents argotiques et les synonymes. En principe, le dictionnaire doit couvrir le droit pénal, la criminologie et la criminalistique de même que les sciences connexes, étant destiné aux spécialistes intéressés par les problèmes criminels sous tous leurs aspects et dans tous les pays. 
En fait, les deux tiers du volume sont réservés aux termes de criminalistique ; ainsi le signalement et l'identification des criminels, les taches et traces, la balistique, les incendies et les faux parce qu'étroitement liés aux sciences exactes et à leurs techniques particulières engendrent un vocabulaire précis dont on traduit tous les détails et toutes les expressions. Reflet d'un passé encore proche où la criminologie naissait? Expression d'une école allemande où la technique a toujours occupé une place prépondérante? Il demeure que l'ouvrage présente aussi d'autres lacunes significatives. Ainsi sur une liste de vingtdeux expressions, sous le mot « crime », figurent les «crimes du grand monde» et «crimes de la haute société » alors qu'on ne retrouve ni le crime économique ni la criminalité d'affaire. Le seul type de sentence retenu est la sentence de mort. Les domaines du traitement des criminels, de la rééducation, de l'assistance post-pénale et de la prévention sont aussi négligés. « Réinsertion sociale », «centres de transition », "foyers d'accueil et de groupe », autant d'omissions qui parlent d'elles-mêmes.

Mais notre propos est moins de faire le procès d'un dictionnaire vieux de vingt ans que de souligner le vieillissement considérable que subissent les termes dans notre discipline.

On allèguera que la disparition et l'avènement de mots est un fait de langue connu. Ce qui est propre aux sciences sociales, cependant, c'est le rythme effréné avec lequel les termes se substituent les uns aux autres. Ainsi la substitution du mot déviance au mot délinquance illustre bien une orientation nouvelle de la pensée. M. D. Szabo en fait état en ces termes :

La déviance est un concept qui englobe la délinquance, résultat d'une technicalité purement juridique et sociologiquement accidentelle. La déviance, elle, se définit par rapport au conformisme ${ }^{1}$.

La naissance dü nouveau concept de déviance témoigne de la distance que l'homme de science contemporain a prise par rapport à l'échelle des valeurs morales De même, le têrme «déjudiciarisation » est né d'un besoin, d'une nouvelle conception de l'administration de la justice. Nous sommes encore loin de nous interroger sur l'admissibilité des néologismes dans le vocabulaire de la langue. L'arrivée massive de concepts neufs exige qu'on invente toujours plu's rapidement des termes pour en rendre compte. Et l'affinement des concepts donne naissance à tout un lexique qui vivra le temps de la pensée qui l'a engendrée. Certains termes se révéleront fertiles et demeureront ; de nombreux autres tomberont en désuétude, après cinq ans, vidés de leur contenu. Les sciences taxonomiques et descriptives n'ont pas ce problème. Il est propre aux disciplines reliées à la morale et à une société qui change constamment les règles du jeu. C'est pourquoi, en criminologie, l'absence de consensus durable sur les concepts fondamentaux empêche toute normalisation des termes et partant, rend très difficile toute tentative pour rassembler un corpus exhaustif, qu'il soit unilingue ou polyglotte.

1. D. Szabo, Criminologie et politique criminelle, Paris, Vrin, 1978 (sous presse). 
Le criminologue est aussi confronté à plusieurs niveaux de langue. Le chercheur qui s'intéresse à l'attitude du public, à sa réaction face à la justice par exemple, et le praticien qui travaille en institution logent à la même enseigne; les propos recueillis, qu'ils fassent l'objet d'une analyse subséquente ou qu'ils servent de moyens de communication ponctuels, exigent de part et d'autre la maîtrise d'un code fort éloigné de celui des théoriciens. Toute pathologie socio-culturelle véhicule des particularismes linguistiques difficilement transcriptibles. Aussi, les lexiques abondent-ils dans plusieurs domaines. Mais il s'agit de glossaires unilingues qui définissent en langue populaire les idiomes propres aux diverses sous-cultures. L'argot carcéral est sans doute le plus connu, pour avoir été récupéré par un certain type de littérature. Qui n'a pas lu Papillon? Comme le jargon des fous et celui des drogués, le vocabulaire des détenus sert à définir leur appartenance et à identifier leur classe ; il recèle des jugements de valeurs à travers un hermétisme significatif. Il reste que le clinicien qui s'y voit confronté doit pouvoir en jouer et en tirer profit et que le chercheur est souvent forcé de le reproduire pour l'analyser et l'interpréter.

L'importance des divers vocabulaires argotiques est apparue au gouvernement américain qui en a réalisé plusieurs traductions en diverses langues au profit des équipes médicales et des troupes séjournant à l'étranger. Malheureusement, il n'en existe pas, à notre connaissance, d'équivalent québécois. Le problème que pose ces «langues marginales» dans un contexte social bilingue s'imagine facilement, mais seul celui qui doit y faire face quotidiennement en mesure toute l'envergure. La traduction d'une simple entrevue ou la rédaction d'un rapport bilingue prend immédiatement des dimensions insoupçonnées. Comment par ailleurs prendre connaissance de telle petite étude qui pourrait être utile si cela requiert au préalable une double traduction ? Il serait intéressant à cet égard de connaître combien d'hommes et combien d'heures ont été consacrés à la transcription et à la traduction française des dialogues contenus dans les rapports de la Commission d'enquête sur le crime organisé !

Des solutions? Sans doute en existe-t-il plusieurs, les unes de type traditionnel, les autres faisant appel aux techniques modernes. Le criminologue dispose déjà de bons lexiques : en psychiatrie, en sciences juridiques, en administration pénitenciaire et en psychologie. Il a l'avantage, au Canada, de pouvoir obtenir des documents bilingues et traduits avec assez de rigueur de la plupart des ministères et des commissions d'enquête.

Sur un autre plan, les banques de terminologie sont appelées à se développer, à devenir de plus en plus accessibles et à se spécialiser. Il en est de même pour le traitement automatique de l'information qui fournit le thesaurus bilingue dont la fonction première est de décrire l'information contenue dans la littérature. Les descripteurs répartis en champs sémantiques, en sous-ensembles et en ensembles sont choisis parmi les termes de pratique courante chez les spécialistes. Un thesaurus ne définit pas les termes mais les présente dans un contexte qui permet d'en préciser le sens. De plus, il se prête facilement à des mises à jour et peut constituer un répertoire fidèle et cumulatif adaptẻ aux besoins des lecteurs. Ainsi le Thesauris pour le traitement de l'information en 
sociologie ${ }^{2}$ peut-il servir de lexique anglais-français et français-anglais, la recherche de chaque mot ou expression étant facilitée par des listes alphabétiques permutées dans chaque langue.

En criminologie, le langage joue un rôle primordial. À travers hui, les concepts s'articulent, les réalités surgissent, dépouillées de leurs contingences particulières, les idéologies parfois contradictoires se dessinent.

La nécessité de pouvoir non seulement traduire mais encore transposer des termes, des concepts et des perspectives d'un cadre culturel à un autre s'accroît avec l'élargissement des champs de la recherche criminologique. En effet, l'Australie, l'Afrique et l'Amérique latine intéressent de plus en plus les centres de recherche américains et européens. La criminologie comparée, longtemps discutée et à bien des égards, s'avère de plus en plus féconde dans des domaines spécifiques, tant en matière de prévention que de traitement.

Peut-être, face à son besoin croissant de communication, le chercheur acceptera-t-il de sacrifier un snobisme qui lui fait souvent adopter un certain style, se montrer « inventif » et «créateur » et modifier le code sans cesse et souvent comme à plaisir ? Avouons que le jargon savant contribue à épuiser bien des tentatives qui permettraient de construire un véritable lexique de base commun à tous les criminologues. Loin de nous la pensée de « geler » l'écriture en criminologie, mais il est clair que les problèmes de traduction dont nous avons discuté ici seraient à moitié résolus si les chercheurs en arrivaient à un consensus sur la décision d'un certain nombre de termes de base.

Que les structures sociales, les modèles culturels et les institutions varient dans le temps et dans l'espace, personne n'y peut rien. Que les idées évoluent, c'est inévitable. Il n'en reste pas moins qu'il est à la fois possible et nécessaire d'arriver à établir des équivalences sémantiques entre les termes de plusieurs langues, équivalences qui permettront de véritables échanges interculturels à tous les niveaux. Cette étape marquera probablement la maturité de la criminologie comme science de l'homme.

JacQueline De Plaen

2. J. Viet, Thesaurus for Information Processing in Sociology/Thesaurus pour le traitement de linformation en sociologie, Paris - La Haye, Mouton, 1971, 336 p. 


\section{BIBLIOGRAPHIE}

ADLER, J.A. (1960), Elsevier's Dictionary of Criminal Science, Amsterdam, Elsevier Publishing Company, $1460 \mathrm{p}$.

CHARRIÈRE, H. (1970), Papillon, Paris, Laffont, 698 p.

HAYS, D.G. (1959), Automatic Language-Data Processing in Sociology, Santa Monica (Calif), Rand Corporation, $32 \mathrm{p}$.

IONESCO, E., COMBET, G. (et al.) (1977), « Six spécialistes en quête d'un bon langage technique 》, la Gazette de la presse de langue française, no 20 , juin-juillet, 6-9.

JONYNAS, A.V. (1975), Bibliographie des sciences sociales, Montréal, Les Presses de I'Université de Montréal, 216 p.

NATIONAL INSTITUTE ON DRUG ABUSE (1975), Operational Definitions in SocioBehavioral Drug Use Research, by J. Elinson et D. Nurco, Rockville (Md.), National Institute on Drug Abuse, 170 p.

NATIONAL INSTITUTE OF LAW ENFORCEMENT AND CRIMINAL JUSTICE (1974), Terms and Definitions for Police Patrol Cars - Law Enforcement Standards Program, by R.G. Massey and W.F. Druckenbrod, Washington (D.C.), Law Enforcement Assistance Administration, $24 \mathrm{p}$.

SANDRY, G., CARRÈRE, M. (1960), Dictionnaire de l'argot moderne, Paris, Éditions du Dauphin, 297 p.

SUMPF, J., HUGUES, M. (1973), Dictionnaire de sociologie, Paris, Larousse, 255 p.

SZABO, D. (1978), Criminologie et politique criminelle, Paris, Vrin (sous presse).

VIET, J. (1971), Thesaurus for Information Processing in Sociology/Thesaurus pour le traitement de l'information en sociologie, Paris - La Haye, Mouton, 336 p. 